\title{
A Review of Skin Melanoma Detection Based on Machine Learning
}

\author{
Ashish Anil. Meshram \\ Tulsiramji Gaikwad-Patil College of Engineering \& Technology Nagpur, India \\ sshishameshram1@gmail.com
}

Anup Gade, Abhimanyu Dutonde

Tulsiramji Gaikwad-Patil College of Engineering \& Technology Nagpur, India

deanacademics@tgpcet.com

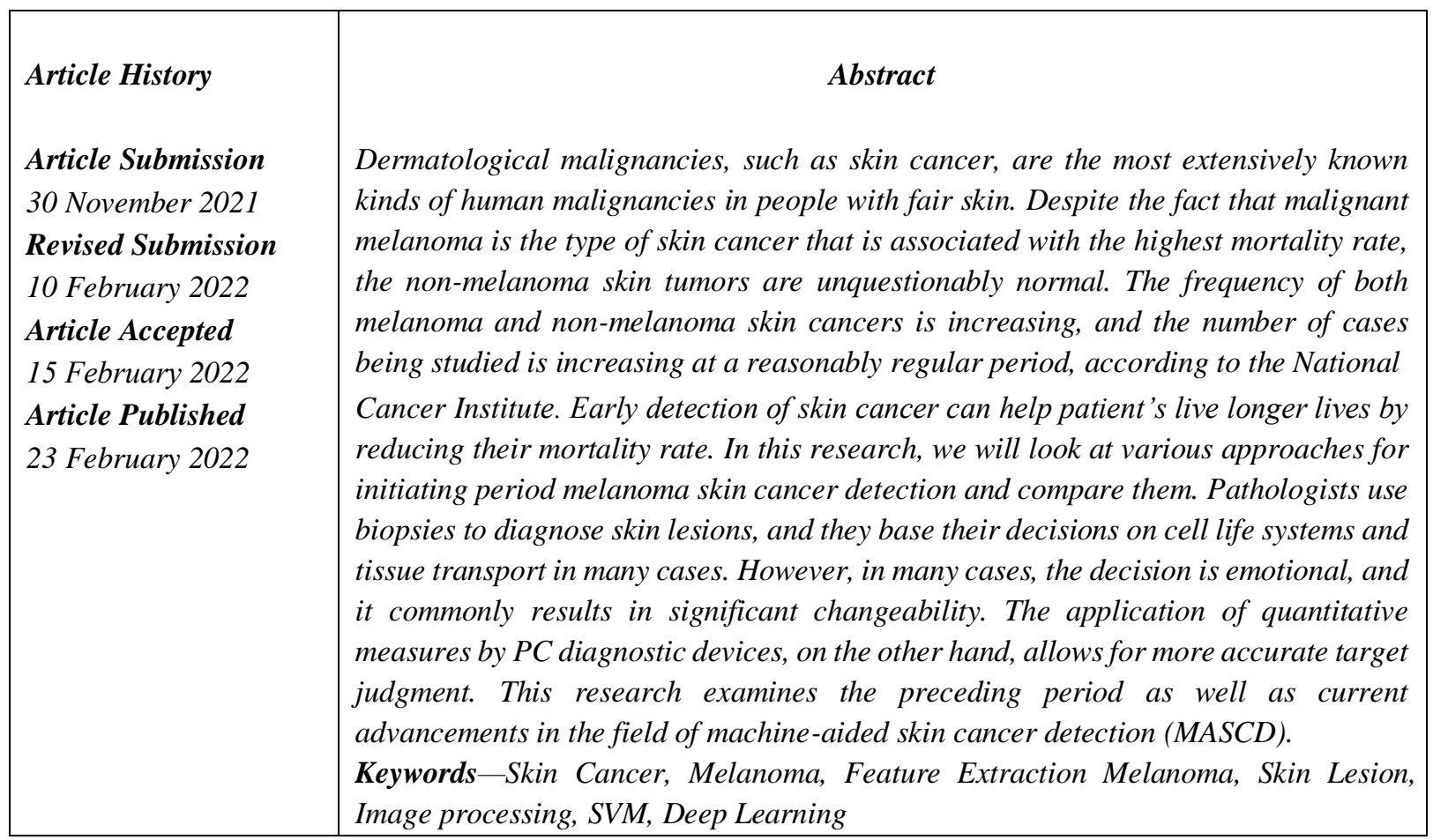

\section{Introduction}

Animals, human beings, and flora are the most not unusual assets of skin cancer in the interim. Dermatological illnesses are a special kind of ailment this is resulting from microscopic organisms or environmental contaminants. The WHO's studies, consistent with an editorial written with the aid of Allison Van Dusen and posted by means of Forbes in July 2008, supplied an information of the causes of pores and skin cancer as well as information at the nations which are disproportionately stricken by the sickness. He looked into information from everywhere in the world, and observed that the countries with the very best consistent with-capita skin cancer issues as a result of ultraviolet (UV) radiation encompass the United States, Canada, and Cuba, in addition to quantities of the Pacific and, most shockingly, Northern Europe, amongst others. While most people of the countries at the list are from South Asia, which includes India, the Maldives, Bhutan, and Bangladesh, the lowest of the listing is ruled through European countries.

According to a report by way of the Skin Cancer Foundation, upwards of 5.4 million cases of nonmelanoma skin most cancers had been treated in greater than 3. Three million human beings within the United States in 2012, the maximum current 12 months for which clean facts become to be had. Every year in the United States, greater human beings are diagnosed with pores and skin cancer than with every other form of most cancers put together. By the age of 70, one in each five Americans may have advanced pores and skin cancer. Actinic keratosis is the maximum appreciably recognized pre-most cancers, affecting greater than 58 million human beings in the United States alone. The cost of treating skin cancers in the United States is anticipated to be $\$ 8.1$ billion per 12 months, with around $\$ 4.8$ billion spent on non - melanoma pores and skin cancers and \$three. 
Three billion spent on melanoma. According to the American Cancer Society's latest statistics, 68, one hundred thirty new cases of melanoma have been discovered inside the United States in 2010, with 8, seven hundred cancer deaths mentioned that yr. (contrasted with eight,420 evaluated passing in 2009). People aged 15- 44 years in Australia are the most liable to cancer, consistent with information. It speaks to ten percentage of all malignancies, which is four times extra than in Canada, the United Kingdom, and the United States, with more than 10,000 times evaluated and approximately 1250 people demise as soon as a year.

Melanoma and nonmelanoma pores and skin cancers are the 2 maximum common kinds of skin most cancers. Malignant cancer and squamous mobile carcinoma are of the maximum famous varieties of pores and skin most cancers. Both are nonmelanomas and can be risky if left untreated. They shape step by step, best now and again spreading past the pores and skin's floor, and are in the end found and, in most instances, restored. Basal cell carcinoma is the maximum common sort of skin most cancers, accounting for about 3 out of every four cases. Melanoma is greater competitive and greater prone to spreading than other kinds of cancer. Kaposi's sarcoma is a rare nonmelanoma this is distinguished via its purple growths and improvement. It is related to a delicate invulnerable framework and has the capability to be extra real. Individuals suffering from AIDS in addition to the elderly are more likely to settlement it. Some noncancerous pores and skin modifications have the capacity to emerge as cancerous. A very well are actinic Kerasotes, that are dried up, red patches on sun-exposed skin that may be scratched off however reappear after some time. Melanoma, the opposite form of skin most cancers, is a potentially deadly and lethal cancer that could unfold quickly. It can originate in stupid skin tissue, such as a mole or pigmentation, and may unfold to greater pigmented pores and skin in addition to non-pigmented skin. Male zits typically start off evolved first on the head, neck, or between the shoulders and hips of the person who is suffering from it. Ladies are more at risk of getting it on the legs and arms than men. Also, feasible places encompass the palm of your hand, the underneath of your foot, beneath this type of fingernail or toenail, physiological fluid linings (consisting of the linings of your mouth, vagina, or buttocks), or even the whites of your eyes. Melanoma isn't always a difficult sickness to treat and is normally curable if caught and handled early. As a result, it progresses more quick than other styles of pores and skin most cancers, and it has the ability to spread past the pores and skin to other regions of the frame, consisting of as the bones and the cerebral cortex. It is tough to deal with and there may be no manner to cast off it at that factor.

Because of the general sustained increase inside the prevalence of melanoma in recent years, as well as its excessive morbidity and mortality and the good-sized person medical cost, its preliminary end has end up a chronic requirement of general fitness. The potential to apprehend cancer, to distinguish malignant systems from favourable and sound systems, and to distinguish the danger level of most cancers are all essential abilties. Generally talking, skin cancer may be divided into two classes: 1) non-cancer and 2) cancer. Melanoma skin cancer (NMSC) and a pair of) Melanoma pores and skin cancer (MSC) are forms of skin cancer (MSC) Early detection and assessment of affected person visibility in skin cancer is the maximum important element to bear in mind.

\section{Image Processing in Medical Science and Skin Cancer Diagnostics}

From the invention of the X-ray by using Wilhelm Roentgen in 1895 through the development of modern-day imaging structures which includes Magnetic Resonance Imaging (MRI) and Computed Tomography (CT), innovation has progressed appreciably. The improvements in imaging innovation will retain to progress as time is going on. In any case, the focus of frameworks is moving far from the therapeutic imaging centre, from the age and acquisition of snap shots, and in the direction of the publish-processing and management of picture records. Because of the necessity to make powerful use of the facts that is already to be had, this turns into even extra important. Recent advances in imaging studies have tested the capacity for innovation to enhance and alter a wide range of components of clinical medicinal drug improvement. Using the example of Biomedical Imaging Systems, Figure 1 illustrates the relationship between the numerous studies regions. Within the sphere of organic picture processing, we are able to see that research is now being conducted on two vital outskirts: a) Image Retrieval Image restoration techniques talk to the gadgets which might be used to search for a specific photograph amongst a huge wide variety of images which are often stored in a database, in preference to a difficult force. As can be mentioned in a few seconds, the gadget in use is either a message assembles or one that is depending on the content material of the picture, relying at the occasions. b) Image Processing 
The image can then be upgraded or recreated, or computerized research may be used to highlight or deliver up zones that can be of significance to the customer after it's been correctly recovered.

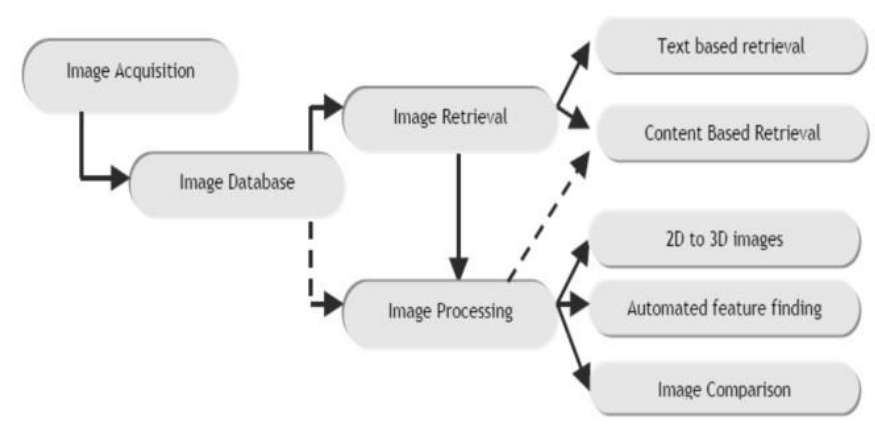

Figure 1. Research Areas in Biomedical Imaging Systems

The application of photo processing to the creation of diagnostics is a non-intrusive technique. There is excellent exhilaration proper now approximately the capability of programmed photograph exam techniques for image processing, each for their capability to provide quantitative information about a lesion, which may be beneficial in the scientific putting, and for his or her ability to function an independent early caution device. In order to gain the remaining aim of growing a successful method of detecting skin most cancers at an early degree without the want for needless pores and skin biopsies, advanced images of melanoma pores and skin lesions were studied in depth. The purpose is to remove the need for useless pores and skin biopsies inside the future.

\section{Proposed System}

In our gadget, we recommend to apply the segmentation, class, inclusive of Convolution Neural Network methods, along with different techniques.

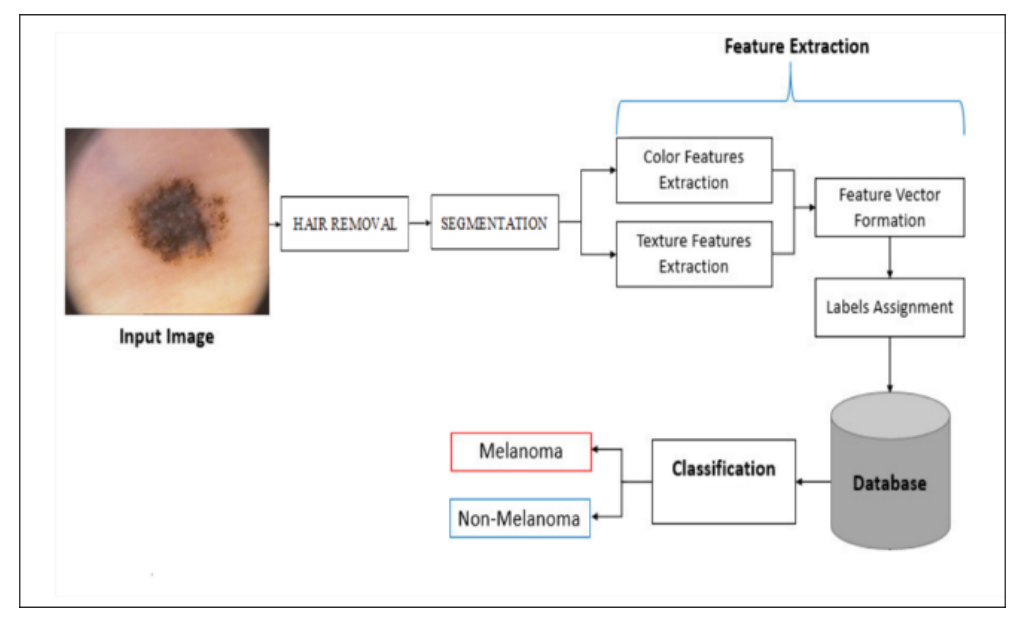

Figure 2. System Architecture

The structure of our counselled gadget is depicted in Figure 2. First and predominant, an image of pores and skin cancer may be supplied as historical past information. In the skin cancer photograph, there are some antiquities to be prevented within the pre-processing, inclusive of hair, which ought to be avoided. The centre channel may be used to discharge hair, that is handy. Pre-processing steps encompass converting an RGB image to a grayscale image, making use of differentiating extending at the photograph, and then performing division on the photo. The extraction of facts is performed within the subsequent stage. The process of extracting highlights is step one in the direction of determining parameters that correspond to the characteristics of the information image, the effects of with the intention to have an effect on the implementation of the characterization framework. The final development is the clustering of lesions. 


\section{Literature Review}

It is crucial to properly diagnose an ambiguous skin lesion so as to administer the essential remedies. The ability to locate cancer early in thermoscopic photographs has a good-sized effect at the survival charge. Only dermatologists who've had widespread training are capable of accurately figuring out melanoma pores and skin lesions. However, the best reputation of cancer is extraordinarily tough because of the accompanying factors, which encompass: minimal differentiation between lesions and skin, visual similitude between malignant and nonmelanoma lesions, and so on. Because expertise is in quick supply, stable programmable detection of pores and skin malignancies, i.e., Frameworks that can be used to organized skin lesions, may be incredibly vital in improving the precision and competency of pathologists, especially in growing nations. In this paper, we supply an outline of the early years of development into cutaneous cancer as well as a method for detecting the sickness.

In their paper [1], Wilson F. Cueva et al. Mounted the premise for the diagnosis of malignant melanomas as a device to provide a second attitude at the identification of this contamination bankruptcy discusses the findings of the $\mathrm{ABCD}$, which provided a excessive level of unshakable first-rate. To obtain asymmetry, border, shade, and diameter, a laptop-based method based totally on photo processing turned into advanced (ABCD of cancer). The use of neurotransmitter receptors to behave out a grouping of different forms of moles is being explored. In a comparable vein, the framework produces consequences with extra performance because the inspection and photograph processing are achieved in quick bursts of time, confined best by the type of $\mathrm{PC}$ and processor this is made to be had to it, ensuing in a greater handy and green output. It changed into located that the calculation built up performed ninety-seven.51 percent of the time after an inquiry of 201 snap shots; at the off danger that we bear in mind an expert (seventy-five to 84 percentage), the framework achieves a excessive degree of productiveness.

Farzam Kharaji Nezhadian et al. [2] confirmed but some other computation to prepare dermoscopic images amongst malignant and beneficent organizations based totally on their findings. The technique is dependent on eye-derivation, and the belief of melanoma within the early tiers is tough for dermatologists to reach. In mild of the truth that non-intrusive units with excessive accuracy and execution had been significantly improved in recent years, structures for picture processing can be used to research and treat illness without the want for invasive techniques. There changed into a purpose to this inquiry, which became to differentiate between benign and malignant melanomas. The potential to section images with high precision was the biggest development. Consequently, a dynamic counter version was used, and the starting area was set with the aid of the purchaser that allows you to boom exactness. Surface - based highlights and RGB components have been used to remove undesirable elements from the picture. The maximum productive detail changed into decided on for the wager lattices of wavelet alternate, which is a TC highlight.

Uzma Bano Ansari et al. [3] proposed a pores and skin most cancers detection framework based totally on assist vector machines (SVM) for the early prognosis of skin cancer sickness. It is more superb to the sufferers. The diagnosing method uses image processing strategies in addition to the calculation of Support Vector Machines (SVM). The dermoscopy image of skin most cancers is taken, and it's far subjected to a spread of pre-processing strategies, such as noise elimination and photo enhancement, earlier than being displayed. Following that, the image is skilled to department via the use of the Thresholding process.

Using the GLCM gadget, it is necessary to put off some highlights from the photograph. These specifics are provided because the classifier's contribution to the overall category. The characterization cause is done through the usage of a bolster vector Machine (SVM). It categorizes the image into categories: cancerous and noncancerous. It was concluded from the evaluation findings that the proposed association of pores and skin cancer detection can be actualized with the help of a dim stage co-occasion framework and an aid vector gadget to quickly distinguish whether or not an image is cancerous or non-cancerous. There is ninety five percent accuracy inside the proposed framework.

Using Image Processing equipment, Shivangi Jain and co-workers [4] evolved a laptop-primarily based method for the identification of Melanoma Skin Cancer that is based totally on image processing. The skin lesion photo is 
furnished as a contribution to the framework, that's then examined using precise photo processing algorithms to decide the likelihood of skin cancer within the vicinity of the skin lesion. The Lesion Image Investigation Instruments examine the surface, size, and shape of the lesion for the presence of various Melanoma parameters such as asymmetry, border, color, diameter, (ABCD), and so on. The photo department and spotlight ranges are done the use of the floor, size, and shape examination. The parameters of the eliminated detail are used to differentiate among everyday pores and skin and Melanoma cancer lesion inside the photo. Following a radical evaluation of the framework, the author concluded that the framework recommended may be used correctly by means of sufferers and clinicians to have a look at skin most cancers in a extra unique way. Especially beneficial in rural regions where experts in the scientific area might not be with ease to be had, this device is extraordinarily flexible.

It has been proposed with the aid of the researchers Suleiman Mustafa et al. [5] that they could use plain snap shots of affected skin places to pick out cancer malignancy. Because of this, creator guarantees that most of the time ABCDEs administer has been linked for the detection of melanoma, and that they connected comparative method as a end result. By utilizing photo processing procedures, they may be able to give attention to a few traits, for example, the shape, shading, and geometry, of a records photograph, which has all the earmarks of being melanoma. Through the use of a support vector gadget with Gaussian spiral premise piece (SVM•RBF), those extricated capabilities are classified as cancerous "malignant" or non-cancerous "beneficent," depending on whether or not they're cancerous or non-cancerous.

They carried out a centered evaluation wherein they attempted numerous things with 2 hundred pix (a hundred of melanoma and 100 of benign) and discovered that only six highlights have been enough to distinguish cancer from benign tissue. There were exams that have confirmed that high precision may be accomplished with a small quantity of precious configurations of capabilities. Particularly, handiest six highlights are enough to distinguish the majority of malignant instances and to offer reliable facts for skin most cancers screening. Larger datasets, however, are required before such frameworks may be used clinically to aid within the recognition of cancer. To aid improvement, testing, and investigation, larger datasets are required.

Furthermore, Deep convolution neural networks (CNNs) display promise for well-known and deep factor allocations across a variety of satisfactory-grained protest classes, consistent with Andre Esteva et al [6]. Using handiest pixels and sickness names as facts assets, the creators of [6] reveal the sequence of skin lesions the usage of a unmarried CNN that has been trained from begin to complete from pix. Immediately after that, they create a CNN the usage of a dataset of 129,450 clinical pics - two requests of more size than previous datasets 12 - that carries 2,032 special infections. Malignant melanomas versus benign seborrheic keratoses where the 2 primary paired order utilize instances that were used within the execution. The execution turned into tried against 21 boardaffirmed dermatologists on biopsy-verified clinical images with two simple paired order utilize cases: keratinocyte carcinomas as opposed to benign seborrheic keratoses; and malignant melanomas versus benign nevi. The first example relates to the identification of the most commonly recognized cancers, even as the second case pertains to the identity of the deadliest kind of pores and skin most cancers. As a end result of those checks, it has been proven that the CNN is capable of retaining up with each single attempted grasp at some stage in the 2 responsibilities, demonstrating a person-made cognizance able to ordering pores and skin most cancers with a stage of skill ability akin to the ones of dermatologists. Cell phones, that are ready with deep neural networks, have the capacity to amplify the reach of dermatologists beyond the confines of the health facility.

In this investigation [7], Yuexiang Li et al. Labored on deep gaining knowledge of strategies, the Lesion Indexing Network (LIN) and the Lesion Feature Network (LFN), to cope with 3 number one obligations, specifically, lesion segmentation, lesion dermoscopic characteristic extraction, and lesion class, all of which might be emerging inside the discipline of pores and skin lesion picture processing. The creator offered a deep getting to know machine that consisted of fully convolutional residual networks (FCRNs), every of which added the department result as well as the coarse characterization end result at the equal time. A lesion index calculation unit (LICU) is created with the intention to refine the coarse grouping consequences by way of figuring out the separation warm guide, that's used within the separation warm manual calculation. For something like the dermoscopic spotlight extraction venture, it's far proposed to use a trustworthy $\mathrm{CNN}$ algorithm. The advised deep learning gadget turned into 
evaluated the usage of the ISIC 2017 dataset, which was provided via the writer. As verified by the proposed (LIN) for lesion division and order, it outperforms the modern deep studying structures, even though the proposed LFN achieves superior ordinary exactness and affectability. The proposed LFN also achieves superior normal exactness and affectability for dermoscopic consist of extraction, demonstrating its notable responsibilities with regards to tending to the take a look at.

The authors of [8] evolved a very programmed approach for skin lesion department that makes most efficient use of a 19-layer convolution neural network (CNN) that does not rely on heritage information of the statistics. This strategy does now not depend upon historical past information of the records. Creator developed a fixed of systems that ensure effective and a success studying even if most effective a limited quantity of guidance knowhow is to be had. A novel misfortune painting primarily based on Jaccard separation is also advanced in an effort to get rid of the requirement for check re-weighting. This is completed as a result of the large inherent unfairness between both the quantity of vanguard and basis pixels whilst using move entropy as that of the misfortune work for picture division, rather than the traditional method. The viability, profitability, and speculating ability of the proposed device have been evaluated the use of two publicly available databases, the ISBI 2016 database and the PH2 database, each of which had been made to be had by means of the author. According to the findings of the writer's investigations, the proposed technique outperformed other high-qualityin-elegance computations on these databases.

This is wherein the paintings of Supriya Joseph and colleagues [9] comes in. They offered a non-intrusive robotized pores and skin lesion observe framework for the early prognosis of melanoma that uses image processing tactics and portable improvements. Hair detection and expelling are carried out with a purpose to ensure that the order is completed correctly, and extraction highlights the pores and skin wound. For the hair ejection, a swift on foot in repainting calculation is hired. It is greater effective for the framework to be effective if the hair that can be gift on the pores and skin is removed. The consequences of the test are in comparison to the $\mathrm{PH} 2$ database from the Pedro Hispano restoration centre.

In this investigation, Lequan Yu et al. [10] proposed another method for cancer popularity that makes use of deep convolutional neural networks (CNNs) and compared it to preceding strategies that employ either lowstage handcreated highlights or CNNs the usage of shallower fashions. Inferred by means of the creators is that their framework, which incorporates extensively deeper networks, can collect more extravagant and discriminative functions for greater particular identification. In order to absolutely gain from deep networks' advantages, the developer proposed an organization of plans that might offer a hit education and mastering in spite of the constraints of confined training information. The following tendencies are incorporated into the approach:

When a device will become greater complex, you may use the leftover understanding to discern out the way to modify to corruption and over fitting problems. It will make certain that the overall performance enhancements achieved through increasing system capability are maintained.

a. In order to attain exact skin lesion department, we will construct a very convolution resting system $($ FCRN) and decorate its skills even in addition through combining it with a multi-scale logical and bodily mixture plot.

b. Finally, merge the recommended FCRN (for division) and deep lingering networks (for arrangement) right into a two-agency structure to shape a -enterprise shape.

This structure enables the characterization machine to do away with more agent and precise highlights primarily based on sectioned effects as opposed to the whole dermoscopy snap shots, consequently reducing the inadequacies of the information instruction manner. The ISBI 2016 Skin Lesion Analysis toward Melanoma Detection Challenge dataset turned into used as a basis for the assessment by way of the inventor.

A deep Siamese CNN (SCNN) layout recommended via Yu-A Chung and associates [11] can be organized the usage of most effective parallel image healthy statistics and is able to mastering photo depictions even as requiring 
much less supervision than previous designs. The key concept manner behind this is the first-rate majority of their methodology in a unmarried regulated convolutional neural network, that is as a long way as is feasible from their authentic method $(\mathrm{CNN})$. Utilizing a publicly to be had multiclass diabetic retinopathy fundus imaging dataset, the creator evaluates the scholarly image portrayals on a project of substance-based therapeutic photo recovery. Because of the test results, it is able to be concluded that the writer's framework, i.E. Deep SCNN, is sort of equal to the maximum current single administered $\mathrm{CNN}$, but requires drastically much less tracking for the duration of the education technique.

Haofu Liao [12] demonstrated an investigation at the feasibility of growing a commonplace pores and skin disorder detection framework using Deep Convolutional Neural Networks (CNN). As soon as viable after beginning the framework, it creates a CNN design the use of 23,000 pores and skin ailment pics from the Dermnet dataset and checks its performance with photographs from both the Dermnet and OLE datasets, that are both skin disease datasets. When examined at the Dermnet dataset, the evaluation outcomes of the framework monitor that the advised gadget may additionally attain as excessive as 73.1 percentage Top-1 exactness and ninety-one.0 percentage Top-five precision. The pinnacle- 1 and pinnacle- 5 correctness's for the take a look at at the OLE dataset are 31.1 percent and 69.5 percent, respectively. A similarly argument positioned out with the aid of the author is that by utilizing greater practice photos, accuracy may be further advanced.

N. C. F. Codella et al. [13] proposed a framework that consolidates ongoing upgrades in deep gaining knowledge of with set up system studying tactics, resulting in companies of techniques that are appropriate for portioning pores and skin lesions and also breaking down the prominent sector and encompassing tissue for melanoma detection, as well as a framework that consolidates ongoing improvements in deep gaining knowledge of with set up gadget studying processes. The system is evaluated using the most important openly to be had benchmark dataset of dermoscopic snap shots, which includes 900 preparing photographs and 379 trying out photographs, respectively. New modern-day execution degrees are verified, resulting in a 7.5 percentage alternate in the territory below recipient operating trademark bend ( 0.843 as opposed to zero.783), a 4-percentage alternate in normal exactness (zero.649 as opposed to zero.624), and a specificity predicted on the clinically extensive 95 percentage affectability running point this is 2.9 times higher than the previous great in elegance (36. Eight percentage specificity contrasted with 12.5 percent).

\section{Analysis and Discussion}

This looks at centerer on Machine Learning Techniques and deep training techniques that mixed CNN with other pre-skilled fashions to categories lesion snap shots. Each set of rules' blessings and disadvantages. The key to getting the fine effects is choosing the right categorization technique. However, CNN affords a advanced outcome than ML methods using pretrained fashions like Alex Net, VGG16, ResNet. Following Table 1 shows the accuracy of various algorithm for skin cancer detection discovered from exclusive researches.

Table 1: Accuracy of Various Algorithms

\begin{tabular}{|c|c|}
\hline Algorithm & Accuracy (\%) \\
\hline SVM & 89 \\
\hline KNN & 79 \\
\hline NB & 86.4 \\
\hline CNN & 91 \\
\hline Region Based CNN & 90.9 \\
\hline Transfer Learning & 93.4 \\
\hline Multiscale CNN & 90.3 \\
\hline
\end{tabular}


Following Figure 3 shows the comparative analysis of the above-mentioned algorithms.

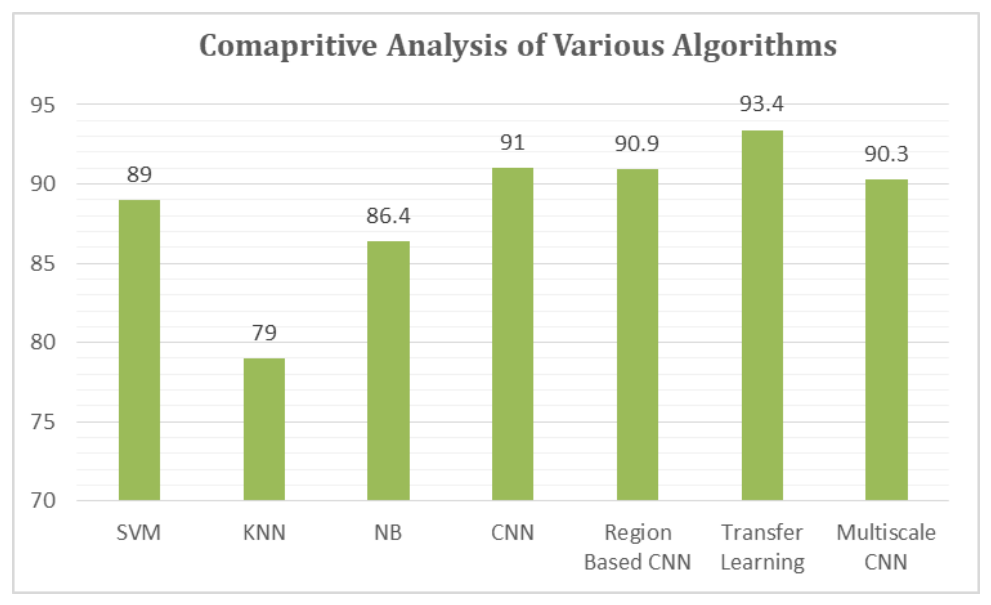

Figure 3. Comparison of Various Algorithms

\section{Conclusion}

Skin cancer detection framework is used to discover and understand skin cancer facet outcomes and finish melanoma in its early levels of development and progression. Following the realization of an investigation into skin cancer detection structures, the introduction of the Computer Aided Diagnosis of the cutting-edge became made. In this review, we study the technological advances that have been made so as to investigate most cancers via the usage of numerous types of images. When in comparison to clinical exam, a combination of photograph coaching and fragile figuring strategies produced more unique outcomes in the detection of cancer than each system by myself. The process of melanoma detection is completed in a number of levels, consisting of preprocessing, division, feature extraction, publish-processing, and course of movement, all of which make use of superior frameworks to provide unique results

\section{References}

[1] Wilson F. Cueva, F. Muñoz, G. Vásquez., G. Delgado, "Detection of skin cancer "Melanoma" through Computer Vision", 2017 IEEE XXIV International Conference on Electronics, Electrical Engineering and Computing (INTERCON), IEEE 2017.

[2] Farzam Kharaji Nezhadian, Saeid Rashidi,"Melanoma skin cancer detection using color and new texture features",2017 Artificial Intelligence and Signal Processing (AISP), IEEE 2017.

[3] Uzma Bano Ansari,Tanuja Sarode,"Skin Cancer Detection Using Image Processing", International Research Journal of Engineering and Technology (IRJET), Volume: 04,Issue: 04, Apr-2017.

[4] Shivangi Jain, Vandana jagtap, Nitin Pise,"Computer aided Melanoma skin cancer detection using Image Processing",International Conference on Intelligent Computing, Communication \& Convergence (ICCC2015), Elsevier - 2015.

[5] Patil, Rashmi, and Sreepathi Bellary. "Machine learning approach in melanoma cancer stage detection." Journal of King Saud University-Computer and Information Sciences (2020).

[6] Patil, Rashmi. "Machine Learning Approach for Malignant Melanoma Classification." International Journal of Science, Technology, Engineering and Management-A VTU Publication 3.1 (2021): 40-46.

[7] Li Y, Shen L. Skin Lesion Analysis towards Melanoma Detection Using Deep Learning Network. Sensors (Basel). 2018 Feb 11;18(2):556. doi: 10.3390/s18020556. PMID: 29439500; PMCID: PMC5855504.

[8] Al-Masni, Mohammed A., et al. "Skin lesion segmentation in dermoscopy images via deep full resolution convolutional networks." Computer methods and programs in biomedicine 162 (2018): 221-231. 
[9] Supriya Joseph, Janu R Panicker,"Skin Lesion Analysis System for Melanoma Detection with an Effective Hair Segmentation Method", IEEE International Conference on Information Science (ICIS), IEEE Aug2016.

[10] Smith, Robert A., et al. "Cancer screening in the United States, 2019: a review of current American Cancer Society guidelines and current issues in cancer screening." CA: a cancer journal for clinicians 69.3 (2019): 184-210.

[11] Yu-An Chung, Wei-Hung Weng, "Learning Deep Representations of Medical Images using Siamese CNNs with Application to Content-Based Image Retrieval",31st Conference on Neural Information Processing Systems (NIPS 2017).

[12] Chintawar, Vedanti, and Jignyasa Sanghavi. "A Review on Computer-Aided Melanoma Skin Cancer Detection using Image Processing." Easy Chair Preprint 584 (2018).

[13] D. Gautam and M. Ahmed, "Melanoma detection and classification using SVM based decision support system,'‘2015 Annual IEEE India Conference (INDICON), 2015, pp. 1-6, doi: 10.1109/INDICON.2015.7443447.

[14] Fei, D.-Y., Almasiri, O., \& Rafig, A. (2020). Skin cancer detection using support vector machine learning classification based on particle swarm Optimization Capabilities. Transactions on Machine Learning and Artificial Intelligence, 8(4), 01-13. https://doi.org/10.14738/tmlai.84.8415

[15] G. Neela Krishna Babu1 and V. Joseph Peter (2021) Skin Cancer Detection Using Support Vector Machine With Histogram Of Oriented Gradients Features, ICTACT Journal On Soft Computing, January 2021, Volume: 11, Issue: 02 Issn: 2229-6956 (Online). Doi: 10.21917/Ijsc.2021.0329

[16] B. Arivuselvam, S. Tanisha, S. Shalini, V.S. Subhalaksmi (2021) Skin Cancer Detection and Classification Using SVM Classifier Turkish Journal of Computer and Mathematics Education Vol.12No.13(2021), 1863-1871 